\title{
Avaliação da dor no recém-nascido prematuro em Unidade de Terapia Intensiva
}

\author{
Pain assessment in the premature newborn in Intensive Care Unit \\ Evaluación del dolor en el recién nacido prematuro en Unidad de Cuidados Intensivos
}

\section{Luciano Marques Santos', Monick Piton Pereira", Leandro Feliciano Nery dos Santos"', Rosana Castelo Branco de Santana ${ }^{\mathrm{IV}}$}

' Universidade Federal do Vale do São Francisco, Grupo de Estudos e Pesquisas sobre Cuidado em Saúde. Juazeiro-BA, Brasil.

"Prefeitura Municipal de São Gonçalo dos Campos. São Gonçalo dos Campos-BA, Brasil.

III Faculdade de Santo Antônio. Alagoinhas-BA, Brasil.

"N Universidade Estadual de Feira de Santana, Curso de Enfermagem. (Graduanda) Feira de Santana-BA, Brasil.

Submissão 08-01-2011 Aprovação: 03-02-2012

\section{RESUMO}

O estudo objetivou analisar o processo de identificação da dor no prematuro pela equipe multiprofissional da Unidade de Terapia Intensiva Neonatal de um hospital público de uma cidade do interior da Bahia. Trata-se de um estudo descritivo, exploratório e quantitativo, realizado com 24 trabalhadores da saúde, através de um formulário. Os dados foram analisados no Statistical Package for Social Sciences. Os resultados evidenciaram que $100 \%$ dos entrevistados acreditavam que o recémnascido sente dor, $83,3 \%$ reconheciam a dor como sinal vital; $58,4 \%$ não conheciam as escalas; $70,8 \%$ não as utilizavam e destacaram sinais fisiológicos e comportamentais como sugestivos de dor. É importante que os profissionais entendam a dor como um fenômeno complexo que demanda intervenção precoce, garantindo a excelência do cuidado.

Descritores: Enfermagem Neonatal; Recém-nascido; Prematuro; Dor; Unidades de Terapia Intensiva Neonatal.

\begin{abstract}
This study aimed to analyze the process of pain identification in premature by the professional staff of the Neonatal Intensive Care Unit of a public hospital in the interior of Bahia, Brazil. This is a quantitative descriptive exploratory study that was made through a form applied to twenty-four health professional of a Neonatal Intensive Care Unit. The data were analyzed in the Statistical Package for Social Sciences. The results showed $100 \%$ of professionals believed that newborns feel pain, $83.3 \% \mathrm{knew}$ the pain as the fifth vital sign to be evaluated; $54,8 \%$ did not know the pain assessment scales; $70.8 \%$ did not use scales and highlighted behavioral and physiological signs of the newborn as signs suggestive of pain. Thus, it is important that professionals understand the pain as a complex phenomenon that demands early intervention, ensuring the excellence of care.
\end{abstract}

Key words: Neonatal Nursing; Infant, Premature; Infant, Newborn; Pain; Neonatal Intensive Care Units.

\section{RESUMEN}

Este estudio tuvo como objetivo analizar el proceso de identificación del dolor en prematuros por el personal profesional de la Unidad de Cuidados Intensivos de un hospital público del interior de Bahía, Brasil. Es un estudio cuantitativo exploratorio descriptivo que se hizo a través de un cuestionario realizado a los veinticuatro profesionales de la salud de la Unidad de Cuidados Intensivos Neonatales. Los datos fueron analizados en el Paquete Estadístico para las Ciencias Sociales. Los resultados mostraron que $100 \%$ de los profesionales cree que los recién nacidos sienten dolor, el $83,3 \%$ conocía el dolor como el quinto signo vital para ser evaluado; $54,8 \%$ no conocía las escalas de valoración del dolor, el $70,8 \%$ no utilizan escalas y destacó fisiológicas signos y de comportamiento signos de los recién nacidos como signos sugestivos de dolor. Por lo tanto, es importante que los profesionales entiendan el dolor como un fenómeno complejo que exige la intervención temprana, garantizando la excelencia de la atención.

Palabras clave: Enfermería Neonatal; Prematuro; Recién nacido; Dolor. Unidad de Terapia Intensiva Neonatal. 


\section{INTRODUÇÃO}

A neonatologia tem passado por profundas transformações nas últimas décadas tanto do ponto de vista tecnológico, quanto da veiculação de evidências científicas que têm proporcionado melhorias significativas no cuidado ao Recém-Nascido Prematuro (RNPT) e sua família.

A sobrevida orgânica do RNPT tem aumentado fazendo com que neonatos com idades gestacionais extremas e/ ou muito baixo peso ao nascimento sobrevivam. Apesar dos avanços tecnológicos e desenvolvimento do país, infelizmente as taxas de prematuridade permanecem elevadas. No Brasil, um país em desenvolvimento, a prevalência de prematuros é de aproximadamente $7 \%{ }^{(1)}$.

Ademais, a diversidade de distúrbios cardiovasculares, respiratórios, metabólicos, congênitos ou cirúrgicos que acometem o RNPT contam hoje com uma tecnologia de ponta e profissionais de saúde capacitados para a implementação de um cuidado cada vez mais complexo e qualificado.

Assim, a necessidade de internação do RNPT em Unidades de Terapia Intensiva Neonatal (UTIN), é de fundamental importância para excluir e/ou amenizar os fatores de riscos determinantes do quadro clínico em que se encontram.

Na UTIN há predomínio de situações adversas decorrentes tanto das normas e rotinas institucionais, quanto do complexo processo de trabalho, as quais contribuem para o desarranjo da homeostasia do organismo do RNPT. Dentre elas, destacam-se o ambiente com luminosidade e temperatura artificial, o barulho incômodo e estressante e ainda, a quantidade de manipulações realizadas nestes pacientes, em geral, agressivas e dolorosas. Desta maneira, ao ser hospitalizado na UTIN, o RNPT fica exposto à realização de técnicas e procedimentos invasivos e potencialmente dolorosos, que poderão impactar em sua qualidade de vida e desenvolvimento neuropsicomotor.

Estímulos dolorosos agudos desencadeiam nos recém-nascidos uma resposta global ao estresse que inclui modificação a nível cardiovascular, respiratório, imunológico, hormonal e comportamental, entre outros. Essas respostas fisiológicas são acompanhadas por uma reação endócrino-metabólica de estresse, com liberação de hormônios como adrenalina, noradrenalina e cortisol, podendo resultar em hiperglicemia e catabolismo protéico lipídico, o que interfere no equilíbrio homeostático, já precário no RNPT ${ }^{(2)}$.

Por conseguinte, o desequilíbrio na fisiologia do organismo pode acarretar a queda na saturação de oxigênio o aumento das frequências cardíaca e respiratória, o estresse, além das conseqüências em longo prazo, tais como o comprometimento do crescimento, desenvolvimento, diminuição do limiar de dor e hiperalgia ${ }^{(3-4)}$.

Um recém-nascido prematuro na UTIN recebe cerca de 130 a 234 manipulações nas 24 horas, sendo que muitas dessas manipulações são dolorosas. Nesta direção, é preciso desconstruir o tradicional argumento de que o RN não é capaz de sentir dor, o qual se apóia na falta de mielinização como um indicador da imaturidade do sistema nervoso central do neonato. É importante lembrar que os impulsos nociceptivos nos adultos também são conduzidos por fibras não mielinizadas e levemente mielinizadas ${ }^{(3,5)}$.

Por isso, negar a existência do processo doloroso no RNPT pode prejudicar a sua avaliação e a intervenção, no contexto da UTIN, principalmente devido a sua característica subjetiva e a necessidade de expressão verbal, o que no cuidado ao RNPT torna-se um dos obstáculos para o tratamento, já que o mesmo não é capaz de expressá-la desta forma. Assim, é essencial a implementação das escalas de avaliação da dor em recém-nascidos, como uma ferramenta clínica de baixo custo e de alto impacto na identificação deste fenômeno.

Os pacientes internados em UTIN são submetidos a vários procedimentos potencialmente dolorosos e o uso da analgesia ainda é pouco freqüente. Há necessidade de aperfeiçoar a formação dos profissionais de saúde para diminuir a distância entre os conhecimentos existentes a respeito do manejo da dor no período neonatal e a prática clínica ${ }^{(6)}$.

Assim, a identificação, avaliação e intervenção no processo doloroso precisam ser uma constante e um desafio a ser superado pela equipe de profissionais envolvidos no cuidado ao RNPT em cuidados intensivos. Porém, apesar dos progressos, nota-se que ainda é escassa a literatura nacional sobre o tema e também há poucos trabalhos relacionados à dor do recém-nascido na área da enfermagem ${ }^{(7)}$. Desta maneira, este estudo teve como objeto de investigação o processo de identificação da dor no RNPT no contexto da UTIN de um hospital público do interior da Bahia.

$\mathrm{O}$ interesse por este objeto de investigação surgiu durante a vivência profissional, ao perceber empiricamente que a equipe de saúde de uma UTIN realizava procedimentos invasivos nos RNPT, sem utilizar instrumentos para a avaliação da ocorrência da dor e/ou para implementar cuidados a fim de aliviar o fenômeno.

Nesta unidade, era constante a manipulação do RNPT, tanto pela equipe médica, quanto pelos enfermeiros, técnicos de enfermagem e fisioterapeutas, para a realização de coleta de material para análise gasométrica, cateterizações venosas centrais e periféricas, sondagens orogátricas, troca de fixações de acessos venosos e sondagens, glicemias capilares, aspiração de vias aéreas, instalação de circuitos de pressão positiva contínua em vias aéreas, dentre outros.

Notou-se que durante a realização destes procedimentos, estes trabalhadores da saúde não consideravam as expressões faciais do RNPT, bem como seu comportamento e respostas fisiológicas como sinais sugestivos de ocorrência da dor, pois a preocupação imediata era com a assertividade do procedimento ou execução do mesmo. Desta forma, a avaliação e a intervenção da dor não era uma constante na prática clínica desta equipe de saúde.

Isto posto, questionou-se: como a equipe multiprofissional de uma Unidade de Terapia Intensiva Neonatal de um hospital público do interior da Bahia realiza o processo de identificação da dor no RNPT?

\section{OBJETIVO}

Analisar o processo de identificação da dor no prematuro pela equipe multiprofissional da Unidade de Terapia Intensiva Neonatal de um hospital público de uma cidade do interior da Bahia. 


\section{MÉTODOS}

Trata-se de um estudo descritivo, exploratório e quantitativo, realizado na Unidade de Terapia Intensiva Neonatal de um hospital público da cidade de Feira de Santana-BA. Esta unidade possui cinco leitos disponíveis a recém-nascidos, em sua maioria, prematuros, e conta com o trabalho de dezesseis técnicos de enfermagem, cinco enfermeiras assistenciais, uma enfermeira coordenadora, cinco médicos e quatro fisioterapeutas, totalizando trinta e um profissionais.

Para a seleção dos participantes deste estudo foram utilizados como critérios de inclusão: ser membro da equipe multiprofissional da UTIN do hospital em estudo; atuar no dia da coleta de dados; atuar no cuidado ao RNPT; aceitar participar do estudo, mediante leitura e assinatura do Termo de Consentimento Livre e Esclarecido. Foram excluídos aqueles que estavam de férias ou licenças e os trabalhadores que desempenhavam alguma atividade administrativa ou burocrática durante o período da coleta dos dados. Desta forma, participaram do estudo 24 profissionais da equipe multiprofissional da Unidade Neonatal em estudo, assim distribuídos: 5 enfermeiras, 2 médicos neonatologistas, 14 técnicos de enfermagem e 3 fisioterapeutas. Foram excluídos deste estudo 7 profissionais devido a ocorrência de férias e licença maternidade.

A coleta dos dados do presente estudo ocorreu no período de janeiro a março de 2009, através de entrevistas estruturadas, sendo utilizado um formulário que continha informações dos profissionais, sobre as escalas de avaliação da dor e as variáveis fisiológicas e comportamentais para a identificação da dor no recém-nascido. Os dados foram analisados no programa estatístico Statistical Package for Social Sciences (SPSS) 15.0. As variáveis categóricas foram expressas por percentual e valor absoluto.

Ressalta-se que o estudo respeitou a Resolução 196/96 do Conselho Nacional de Saúde, sendo aprovado pelo Comitê de Ética em Pesquisa da Faculdade de Tecnologia e Ciências, campus de Salvador-BA, sob o parecer de número 0484-2008.

\section{RESULTADOS E DISCUSSÃO}

Com relação ao reconhecimento da dor, de acordo com a tabela 1, 100\% (24) dos participantes deste estudo referiram que acreditavam no fato do recém-nascido prematuro sentir este processo. Este dado também foi encontrado em estudos nacionais $^{(5,8-9)}$.
Com relação ao reconhecimento da dor como o quinto sinal vital, conforme Tabela 1, evidenciou-se que 83,3\% (20) dos colaboradores deste estudo consideravam a dor como o quinto sinal vital a ser reconhecido no RNPT, enquanto que $16,7 \%(04)$ não a consideravam como uma variável a ser avaliada em conjunto com outros parâmetros clínicos pela equipe multiprofissional.

O não reconhecimento da dor como uma variável vital a ser avaliada na prática clínica diária é preocupante, pois diante do atual estado da arte da produção do conhecimento relativo à dor no período neonatal, nota-se um contingente de profissionais que poderão contribuir com ocorrência de iatrogenias no cuidado ao prematuro, haja vista a possibilidade de realização de procedimentos invasivos e potencialmente dolorosos sem as devidas intervenções para o alívio do processo doloroso.

Assim, nos últimos anos, importantes avanços ocorreram em relação à avaliação da dor com a validação de critérios objetivos, que hoje podem ser utilizados em diferentes locais. Foi de especial importância a padronização da dor como quinto sinal vital, pela Joint Commission on Accreditation of Healthcare Organizations (JCAHO). Esta comissão passou a considerar prioritárias a avaliação, a intervenção e a reavaliação da dor no processo de qualificação hospitalar. Para a JCAHO, a avaliação da dor inclui a localização, a intensidade da dor baseada em escalas comportamentais, parâmetros fisiológicos entre outras ${ }^{(10)}$.

Ao serem questionados sobre o conhecimento das escalas de avaliação da dor, de acordo com a tabela 1, 58,4\% (14) dos profissionais atuantes na UTIN afirmaram não ter conhecimento a respeito da existência destes instrumentos, sendo que apenas 41,6\% (10) conheciam alguma escala utilizada para avaliação deste processo.

Num estudo realizado nesta mesma cidade e nas unidades neonatais da maternidade municipal, os profissionais reconheceram a importância da avaliação da dor nos recém-nascidos prematuros hospitalizados. Entretanto, os entrevistados não utilizavam escalas que subsidiassem esta avaliação, além do fato de não haver uma política institucional e a discussão da dor a nível setorial, como um dos parâmetros vitais, contemplados em protocolos assistenciais ${ }^{(11)}$.

Este achado também foi apontado em uma dissertação de mestrado realizada na cidade de Salvador-BA, com profissionais de UTIN, onde poucos entrevistados demonstraram conhecer a

Tabela 1 - Distribuição do reconhecimento da dor como o quinto sinal vital, do conhecimento e utilização das escalas de avaliação da dor pelos profissionais integrantes da equipe multiprofissional de uma Unidade de Terapia Intensiva Neonatal do interior da Bahia. FSA-BA (2009).

\begin{tabular}{lcc}
\hline \multicolumn{1}{c}{ VARIÁVEIS } & Sim & Não \\
\cline { 2 - 3 } & $\mathbf{F ( \% )}$ & $\mathbf{F}(\%)$ \\
\hline Reconhecimento que o RNPT sente dor & $24(100)$ & - \\
Conhecimento sobre a dor como o quinto sinal vital & $20(83,3)$ & $04(16,7)$ \\
Conhecimento das escalas de avaliação da dor & $14(58,4)$ & $10(41,6)$ \\
Utilização das escalas de avaliação da dor & $07(29,2)$ & $17(70,8)$ \\
\hline
\end{tabular}


existência de escalas para dor, sendo que estes não utilizavam este recurso clínico para o manejo da dor no $\mathrm{RNPT}^{(12)}$.

Estas escalas são utilizadas como instrumentos para percepção da dor principalmente em recém-nascidos, que não podem expressar verbalmente o que sentem, nem tão pouco a intensidade. Estes instrumentos facilitam a interação e comunicação entre os membros da equipe de saúde, que passam a atender e a perceber a evolução da dor em cada paciente e verificar a resposta frente à terapia(10).

O fato de a dor ser um fenômeno subjetivo gera uma grande dificuldade para a elaboração de um método único de avaliação e de fácil aplicação na prática clínica dos profissionais das unidades neonatais. Destarte, há evidências científicas de que o recém-nascido apresenta um modo característico de exprimir a dor, através de uma linguagem própria, sendo importante neste processo a implementação das escalas ${ }^{(13)}$.

Estes achados são reforçados na tabela 01, ao ser evidenciado que $70,8 \%$ (17) dos profissionais do campo empírico em estudo não utilizavam escalas para a avaliação da dor. Apesar de os profissionais de saúde acreditarem que os recém-nascidos sentem dor, sua avaliação é realizada de acordo com valores individuais, sem padronização ${ }^{(12)}$.

Sendo assim, pode-se considerar que o processo de reconhecimento da dor nos neonatos prematuros na referida UTIN vem ocorrendo de maneira individualizada e não sistematizada, mas embasada em valores de formação profissional, o que pode contribuir para algum tipo de repercussão na clínica desta clientela e propiciar o potencial de ocorrência de iatrogenias no cuidado, infringindo os princípios da segurança do paciente.

$\mathrm{Na}$ literatura nacional, referente à identificação do fenômeno da dor, não há consenso sobre a melhor escala a ser utilizada. Por outro lado, são escassos os estudos referentes à utilização de instrumentos para estudo deste processo no RNPT.

Conforme Tabela 2, tanto os trabalhadores da saúde de nível superior, quanto os profissionais de nível médio utilizam sinais fisiológicos como principais parâmetros clínicos na identificação da dor no neonato prematuro de forma empírica, não sendo considerados estes sinais em conjunto como sugestivos deste processo, já que eles não fundamentam a avaliação com base em escalas compatíveis com o período neonatal.

Em relação aos trabalhadores de nível superior, 28,6\% (10) apontaram a taquicardia como sinal sugestivo de dor, 25,7\% (09), a diminuição da saturação de oxigênio e 22,9\% (08), a taquipnéia. Dos trabalhadores de nível médio, 37,9\% (11) identificaram a taquicardia, 24,1\% (07), a taquipnéia e 17,2\% (05), a diminuição da saturação de oxigênio. Estes dados foram semelhantes aos encontrados em outros estudos realizados no Brasil $^{(5,9,14-15)}$.

A dor ativa mecanismos compensatórios do sistema nervoso autonômo produzindo respostas que incluem alterações das frequências cardíaca e respiratória, pressão arterial, saturação de oxigênio, vasoconstrição periférica, sudorese, dilatação de pupila e aumento da liberação de catecolaminas e hormônios adrenocorticosteróides. Porém, a variação desses parâmetros pode não estar relacionada especificamente com um estímulo doloroso, podendo ser por eventos adversos como fome, choro, algum tipo de desconforto, ansiedade ou alterações causadas pela própria doença de base. Devido aos mecanismos de acomodação e adaptação que ocorrem com as respostas fisiológicas, essas variações têm sido mais úteis no exame de experiências dolorosas associadas apenas a procedimentos de curta duração ${ }^{(16)}$.

Por outro lado, estes dados podem relacionar-se às condições clínicas do prematuro, tendo em vista a diversidade de patologias respiratórias que pode acometê-los, os tornando responsáveis pela maior ocupação dos leitos intensivos neonatal. Por outro lado, podem configurar um estado de estresse associado às condições ambientais e à manipulação por parte da equipe multiprofissional.

Nesta direção, ao considerarem isoladamente estes sinais fisiológicos como indicadores da dor no RNPT, estes trabaIhadores da saúde poderão adotar de maneira indiscriminada, estratégias tais como o uso de fármacos para o alívio deste fenômeno, o que poderá prejudicar a sobrevida orgânica do RNPT.

\section{Tabela 2 - Distribuição dos sinais fisiológicos utilizados para a identificação da dor no recém-nascido prematuro pelos profissionais integrantes da equipe multiprofissional de uma Unidade de Terapia Intensiva Neonatal do interior da Bahia. FSA-BA (2009).}

\begin{tabular}{|c|c|c|}
\hline VARIÁVEIS & $\begin{array}{c}\text { NÍVEL SUPERIOR } \\
\text { F(\%) }\end{array}$ & $\begin{array}{c}\text { NÍVEL MÉDIO } \\
\text { F(\%) }\end{array}$ \\
\hline Taquicardia & $10(28,6)$ & $11(37,9)$ \\
\hline Taquipnéia & $08(22,9)$ & $07(24,1)$ \\
\hline Diminuição da saturação $\mathrm{O}_{2}$ & $09(25,7)$ & $05(17,2)$ \\
\hline Dispnéia & $02(5,7)$ & $03(10,3)$ \\
\hline Apnéia & $02(5,7)$ & $01(3,5)$ \\
\hline Aumento da saturação $\mathrm{O}_{2}$ & $02(5,7)$ & $01(3,5)$ \\
\hline Bradicardia & $02(5,7)$ & $01(3,5)$ \\
\hline
\end{tabular}


Tabela 03 - Distribuição dos sinais comportamentais utilizados para a identificação da dor no recém-nascido prematuro pelos profissionais integrantes da equipe multiprofissional de uma Unidade de Terapia Intensiva Neonatal do interior da Bahia. FSA-BA (2009).

\begin{tabular}{|c|c|c|}
\hline & NÍVEL SUPERIOR & NÍVEL MÉDIO \\
\hline VARIÁVEIS & $F(\%)$ & $F(\%)$ \\
\hline Choro & $10(12,0)$ & $14(17,9)$ \\
\hline Movimento dos braços & $09(10,9)$ & $12(15,4)$ \\
\hline Movimento das pernas & $09(10,9)$ & $12(15,4)$ \\
\hline Olhos espremidos & $09(10,9)$ & $11(14,1)$ \\
\hline Fronte Saliente & $08(9,6)$ & $08(10,2)$ \\
\hline Tremor no queixo & $07(8,4)$ & $08(10,2)$ \\
\hline Fenda palpebral estreitada & $07(8,4)$ & $07(9,0)$ \\
\hline Língua tensa & $06(7,2)$ & $02(2,6)$ \\
\hline Protusão de língua & $06(7,2)$ & - \\
\hline Boca aberta & $05(6,0)$ & $02(2,6)$ \\
\hline Sulco nasolabial aprofundado & $04(4,8)$ & - \\
\hline Boca estirada & $03(3,6)$ & $02(2,6)$ \\
\hline
\end{tabular}

Como mostra a Tabela 3, dos profissionais de curso superior que identificaram a dor no recém-nascido através de sinais comportamentais, $12 \%$ (10) consideravam o choro como principal sinal da existência de dor no RNPT, 10,9\%(09) acreditavam que os movimentos das pernas, braços e olhos espremidos indicam sinais de presença do processo doloroso e $9,6 \%(08)$, a fronte saliente. Dentre os profissionais de nível médio, 17,9\%(14) consideravam o choro, $15,4 \%$ (12), o movimento das pernas e braços e 14,1\%(11), o espremer dos olhos. Estes mesmos parâmetros são relatados em pesquisas realizadas no Brasil( $^{(5,9,14-15)}$.

Na Tabela 3, percebe-se que tanto os profissionais de nível superior quanto os de nível médio utilizam os mesmos parâmetros comportamentais na identificação da dor no RNPT, sendo destacados o choro, os movimentos dos membros, os olhos espremidos e a fronte saliente.

A equipe de enfermagem avalia a dor, principalmente, observando mudanças no comportamento da criança, incluindo o choro característico, alterações na mímica facial, no humor e nos movimentos corporais ${ }^{(14)}$.

O choro é uma das manifestações que o recém-nascido utiliza para comunicar sua dor. A emissão de dor do recém-nascido é tensa e estridente, com frequência fundamental aguda e variações encontradas no traçado espectrográfico, como quebras, bitonalidade e frequência hiperaguda. Tais características tornam o choro de dor peculiar, auxiliando na avaliação de dor durante um procedimento e na conseqüente opção por diferentes técnicas e procedimentos a fim de aliviar o processo doloroso dos recém-nascidos ${ }^{(17)}$.
As alterações da mímica facial não trazem informações fidedignas no que diz respeito à qualidade ou à intensidade da dor. Além disso, sabe-se que há alterações aos estímulos dolorosos agudos, mas não se conhece o que ocorre diante de um estímulo prolongado ou repetitivo ${ }^{(18)}$.

O estado comportamental do neonato no momento que antecede o estímulo doloroso afeta a intensidade da resposta. Recém-nascidos em sono profundo demonstram menos dor quando são analisadas as alterações de mímica facial em relação àqueles que estão em estado de alerta. O meio ambiente também interfere na intensidade da resposta ao estímulo doloroso. Por isso, o ambiente deve ser tranqüilo, sem muitos ruídos, com baixa luminosidade promovendo o máximo de conforto possível ${ }^{(16)}$.

Diante de um estímulo doloroso, os recém-nascidos apresentam flexão e extensão dos membros. Essa resposta organizada parece estar presente mesmo nos prematuros, comprovando que existe uma linguagem corporal em resposta ao estímulo de dor no recém-nascido, porém não deve ser utilizado isoladamente como parâmetro para sua avaliação(18).

Neste sentido, os dados apontam para o fato de que os profissionais de nível médio apresentaram maiores frequências em relação à utilização dos parâmetros comportamentais, o que de fato pode ser considerado como um indicador na implementação de estratégias para a educação permanente neste setor, já que são os técnicos de enfermagem que permanecem à beira leito nas vinte e quatro horas coletando informações relativas aos sinais vitais dos recém-nascidos ou realizando alguns procedimentos invasivos e potencialmente dolorosos 
como as glicemias capilares, punções venosas periféricas e a administração de fármacos intravenosos.

Os dados das Tabelas 2 e 3 apontam para a necessidade de estruturação e sistematização da prática clínica da equipe multiprofissional da unidade em estudo, tendo em vista o fato de que esses profissionais realizam a identificação da dor no RNPT com base em variáveis comportamentais e fisiológicas isoladas, já que eles não utilizam escalas apropriadas.

Em estudo realizado na UTIN do Hospital Universitário Regional de Maringá-PR, foi identificada a vulnerabilidade no conhecimento dos profissionais de enfermagem que atuavam nesta unidade neonatal no que se refere à dor e analgesia do recém-nascido, essencial à compreensão da necessidade de identificar e tratar esse fenômeno tão complexo, embora identifiquem e utilizem estratégias para o seu controle ${ }^{(5)}$.

Por outro lado, as alterações comportamentais são consideradas mais específicas do que as fisiológicas quando do reconhecimento do processo doloroso. Faz-se necessário o reconhecimento do contexto global da hospitalização do recém-nascido na UTIN, haja vista a complexidade dos sinais emitidos pelo mesmo diante da variabilidade de condições patológicas associadas a sua imaturidade orgânica.

Assim, considera-se que esta prática pode contribuir para o aparecimento de iatrogenias do período neonatal, as quais favorecem as comorbidades associadas aos distúrbios respiratórios e cardiovasculares, característicos deste período.

Porém, ao se considerar que identificar e aliviar a dor são questões que fazem parte do cuidado, percebe-se que a identificação e o manejo da dor, envolvem questões éticas, bioéticas e humanitárias do exercício profissional. São necessárias mudanças de paradigma com relação a estes processos, os quais devem ser vistos sob o enfoque científico, crítico e reflexivo, considerando toda a complexidade de que se reveste, para que possa ser reproduzido o cuidado e não apenas uma técnica ${ }^{(19)}$.

\section{CONCLUSÕES}

A partir dos resultados obtidos, percebe-se que há pouco conhecimento a respeito da avaliação a ser realizada para a detecção da dor no RNPT internado na UTIN.

Foi observado que, apesar de a maior parte dos trabalhadores da saúde considerar a dor como o quinto sinal a ser avaliado constantemente na prática clínica, e embora conhecessem as escalas específicas para avaliação do processo doloroso no RNPT, em geral, não as utilizavam no seu dia-a-dia profissional.

Notou-se que a avaliação da dor ocorria de forma não sistematizada, já que os entrevistados informaram que utilizavam sinais fisiológicos e comportamentais de forma fragmentada, não considerando o aspecto integral do cuidado para a avaliação da dor no RNPT de acordo com um contexto mais amplo.

Neste sentido, é importante que os profissionais entendam a fisiopatologia, as manifestações clínicas do neonato, o tipo e as características da dor no RNPT, tomando-as como parâmetros para a avaliação clínica e prosseguir com a conduta adequada para amenizar e ou excluir as estimulações geradoras de processos dolorosos neste recém-nascido.

Isto posto, faz-se necessária a educação permanente desta equipe multiprofissional, no tocante à avaliação da dor no período neonatal, destacando as escalas disponíveis, a fim de construir um protocolo assistencial pautado em evidências científicas, garantindo a excelência do cuidado, a segurança do paciente.

Assim, este estudo poderá contribuir para a reflexão crítica dos profissionais envolvidos no cuidado ao RNPT, sobre as ações prestadas na UTIN, incentivando-os a buscar novos métodos para avaliar e diagnosticar a dor do RNPT de forma a nortear a assistência prestada de maneira eficiente e humanizada.

\section{REFERÊNCIAS}

1. Araújo DMR, Pereira NL, Kac G. Ansiedade na gestação, prematuridade e baixo peso ao nascer: uma revisão sistemática da literatura. Cad de Saúde Pública 2007; 23(4):747-756.

2. Guinsburg R. Dor no recém-nascido: importância do estudo da dor no recém-nascido. In: Rugolo LMS. Manual de neonatologia. Sociedade de Pediatria de São Paulo. Departamento de Pediatria. Rio de Janeiro: Revinter; 2000. p.63-9.

3. Costa HPF, Tadeu S. O Recém - Nascido de Muito Baixo Peso. São Paulo: Editora Atheneu; 2004.

4. Gaspardo CM, Linhares MBM. Dor em neonatos pré-termo em Unidades de Terapia Intensiva Neonatal: avaliação e intervenção com sacarose. São Paulo. Dissertação. [Mestrado em saúde mental] - Faculdade de Medicina de Ribeirão Preto, Universidade de São Paulo; 2006.

5. Veronez M, Corrêa, DAM. A dor e o recém-nascido de risco: percepção dos profissionais de enfermagem. Cogitare Enferm 2010;15(2):263-70.
6. Prestes ACY, Guinsburg R, Balda RCX, Marba STM, Rugolo LMSS, Pachi PR, et al. Frequência do emprego de analgésicos em unidades de terapia intensiva neonatal universitárias. J pediatr 2005;81(5):405-410.

7. Lemos NRF, Caetano EA, Marques SM, Moreira DS. Management of pain in the newborn: literature review. Rev enferm UFPE on line [periódicos na internet]. 2010 [acesso em 17 dez 2010]; 4(n. ${ }^{\circ}$ esp.):32-9. Disponível em: http://www.ufpe.br/revistaenfermagem/index.php/revista/ article/view/722

8. Sousa BBB, Santos MH, Sousa FGM, Gonçalves APF, Paiva SS. Avaliação da dor como instrumento para o cuidar de recém-nascidos pré-termo. Texto \& contexto enferm 2006; 15 (n. ${ }^{\circ}$ esp.):88-96

9. Crescêncio EP, Zanelato S, Leventhal LC. Avaliação e alívio da dor no recém-nascido. Rev Eletr Enf [periódicos na internet]. 2009 [acesso em 30 nov 2010];11(1):64-9 Disponível em: http://www.fen.ufg.br/revista/v11/n1/ v11n1a08.htm 
10. Viana DL, Dupas G, Pedreira MLG. A avaliação da dor da criança pelas enfermeiras na Unidade de Terapia Intensiva. Pediatr 2006;28(4):251-261.

11. Ribeiro IS. Cuidado de enfermagem no manejo da dor no recém-nascido prematuro. Feira de Santana. Monografia Faculdade de Tecnologia e Ciências; 2009.

12. Calasans MTA. A dor no recém-nascido no cotidiano da Unidade de Terapia Intensiva Neonatal. Salvador. Dissertação [Mestrado em cuidar em Enfermagem] - Universidade Federal da Bahia; 2006.

13. Balda RCX, Guinsburg R. Avaliação da dor no período neonatal. In: Kopelman BI, Santos AMN, Goulart AL, Almeida MFB, Miyoshi MH. Diagnóstico e Tratamento em Neonatologia. São Paulo: Atheneu; 2004. p.577-585.

14. Oliveira RM, Silva AVS, Chaves EMC, Sales NC. Avaliação comportamental e fisiológica da dor em recém-nascidos pelos profissionais de enfermagem. REME Rev Min Enferm 2010;14(1):19-24.

15. Scochi CGS, Carletti M, Nunes R, Furtado MCC, Leite AM. A dor na Unidade Neonatal sob a perspectiva dos profissionais de enfermagem de um hospital de Ribeirão Preto-SP. Rev Bras Enferm 2006; 59(2):188-94.

16. Silva YP, Gomez RS, Maximo TA, Silva AC. Avaliação da dor em neonatologia. Rev Bras Anestesiol. [periódicos na internet]. 2007 [acesso em 8 jan 2010];57(5):565-574 Disponível em: http://www.scielo.br/scielo.php?script =sci arttext\&pid $=$ S003470942007000500012\&lng $=$ en\&nrm $=$ iso\&tlng $=$ pt.

17. Branco A, Fekete SMW, Rugolo LMSS, Rehder MI. Valor e variações da frequência fundamental no choro de dor de recém-nascidos. Rev CEFAC 2006;8(4):529-35.

18. Guinsburg R. Arias MCC. A Linguagem da dor no recém-nascido. São Paulo: Sociedade Brasileira de Pediatria; 2010.

19. Antunes JCP, Nascimento MAL, Gomes AVO, Araujo MC. Installation CPAP nasal - identifying the pain of newborns as a nursing care. Rev Enferm UFPE On Line [periódicos na internet]. 2010 [acesso em 20 dez 2010];4(1):137-44 Disponível em: http://www.ufpe.br/revistaenfermagem/ index.php/revista/article/view/592 